\title{
Criptógamos do Parque Estadual das Fontes do Ipiranga, São Paulo, SP. Algas 34: Chlorophyceae (Golenkiniaceae e Micractiniaceae)
}

\author{
Andrea Tucci ${ }^{1,4}$, Natali dos Reis Moura Bento ${ }^{1,2}$, Camila Rosal ${ }^{1,3}$ e Carlos Eduardo de Mattos Bicudo \\ Recebido: 23.08.2012; aceito: 27.01.2014
}

\begin{abstract}
Cryptogams of the Parque Estadual das Fontes do Ipiranga, São Paulo, SP. Algae 34: Chlorophyceae (Golenkiniaceae e Micractiniaceae)). Taxonomic survey of representatives of families Golenkiniaceae and Micractiniaceae in the Parque Estadual das Fontes do Ipiranga aquatic systems. Two genera of Golenkiniaceae (Golenkinia and Polyedriopsis) were identified, each with a single species, and two other genera (Golenkiniopsis and Micractinium) of Micractiniaceae, being three of Micractinium and two of Golenkiniopsis. Micractinium was the best represented genus taxonomically, with three species: M. bornhemiense, M. crassisetum and M. pusillum. Garças Pond was the system that presented the highest number of species, including representatives of all species presently identified.
\end{abstract}

Keywords: Brazil, Chlorophyceae, floristic survey

RESUMO - (Criptógamos do Parque Estadual das Fontes do Ipiranga, São Paulo, SP. Algas 34: Chlorophyceae (Golenkiniaceae e Micractiniaceae)). Levantamento florístico das Golenkiniaceae e Micractiniaceae que ocorrem nos ambientes aquáticos do Parque Estadual das Fontes do Ipiranga. Dois gêneros de Golenkiniaceae (Golenkinia e Polyedriopsis) foram identificados, cada um com uma espécie; e dois gêneros (Golenkiniopsis e Micractinium) de Micractiniaceae, incluindo três espécies de Micractinium e duas de Golenkiniopsis. Micractinium foi o gênero taxonomicamente melhor representado, com três espécies: M. bornhemiense, M. crassisetum e M. pusillum. O Lago das Garças foi o ambiente em que se obteve o maior número de registros, por apresentar representantes de todas as espécies ora identificadas.

Palavras-chave: Brasil, Chlorophyceae, levantamento florístico

\section{Introdução}

Golenkiniaceae (Koršikov) Komárek e Micractiniaceae G.M. Smith são duas famílias da ordem Chlorococcales sensu latu, classe Chlorophyceae que possuem registros para as águas doces brasileiras (Bicudo \& Menezes 2006).

Com a biologia molecular, diferentes arranjos vêm sendo propostos na sistemática e nas relações filogenéticas entre os grupos de algas, especialmente para as algas verdes. Krienitz et al. (2003) incluíram as famílias Golenkiniaceae e Micractiniaceae em Sphaeropleales, as quais compreendem as clorófitas unicelulares, filamentosas ou cenobiais, e produzem células móveis biflageladas com corpos basais diretamente opostos. Estes e outros estudos moleculares (ex. Hegewald et al. 2001, Buchheim et al. 2005, Pröschold et al. 2010, Krienitz \& Bock 2012, Leliaert et al. 2012) vêm apresentando uma nova imagem da filogenia das algas em geral, cujos resultados indicam forte antagonismo com os critérios morfológicos aplicados na taxonomia e sistemática tradicionais (Comas \& Sánchez 2008).

Embora a filogenia das algas verdes esteja em amplo desenvolvimento, no Brasil estes estudos são ainda incipientes e o conhecimento sobre este grupo de algas está contido em trabalhos taxonomicos tradicionais. Assim, Sant'Anna (1984) realizou o levantamento das Chlorococcales do Estado de São Paulo e identificou 118 táxons distribuídos em 41 gêneros; as espécies das duas famílias em pauta que constaram nesse levantamento são: Golenkinia radiata Chodat, Golenkinia paucispina West \& West, Micractinium bornhemiense (Conrad) Koršikov,

1. Instituto de Botânica, Caixa Postal 68041, 04045-972 São Paulo, SP, Brasil

2. Programa de Iniciação Científica do Instituto de Botânica de São Paulo, PIBIC/CNPq

3. Programa de Pós-Graduação em Biodiversidade Vegetal e Meio Ambiente do Instituto de Botânica

4. Autor para correspondência: atuccic@ig.com.br 
M. pusillum Fresenius, Phythelios viridis Frenzel e Polyedriopsis spinulosa (Schmidle) Schmidle.

Vários trabalhos relacionados a microalgas planctônicas e perifíticas foram realizados com materiais coletados dos sistemas aquáticos situados no Parque Estadual das Fontes do Ipiranga (PEFI) e abrangeram representantes das famílias Golenkiniaceae e Micractiniaceae, três dos quais versam sobre taxonomia (Sant'Anna et al. 1989, Ferragut et al. 2005, Tucci et al. 2006) e nove sobre ecologia (Sant'Anna et al. 1997, Tucci \& Sant'Anna 2003, Crossetti \& Bicudo 2005, 2008, Lopes et al. 2005, Fonseca \& Bicudo 2008, 2011, Gentil et al. 2008, Bicudo et al. 2009). Nos trabalhos de cunho ecológico, as espécies e variedades taxonômicas dos representantes das referidas duas famílias constaram nas listas dos materiais identificados sem, entretanto, se dispor de descrição e/ou ilustração dos espécimes identificados.

Dos trabalhos exclusivamente taxonômicos realizados no PEFI, Sant'Anna et al. (1989) identificaram a comunidade fitoplanctônica do Lago das Garças e relacionaram as quatro espécies seguintes: Golenkinia radiata Chodat, Micractinium bornhemiense (Conrad) Koršikov, M. crassisetum Hortobágyi e M. pusillum Fresenius; Ferragut et al. (2005) registraram a presença de Golenkinia radiata Chodat no Lago do IAG; e Tucci et al. (2006) descreveram e ilustraram, ao complementarem o inventário florístico da comunidade fitoplanctônica do Lago das Garças, Golenkiniopsis longispina (Koršikov) Koršikov e G. solitaria (Koršikov) Koršikov.

Por ser um levantamento florístico, foi utilizado no presente trabalho o conceito morfológico de espécie considerando os critérios de Komárek \& Fott (1983). Assim, os objetivos desta contribuição foram: 1) realizar o levantamento taxonômico dos gêneros e espécies classificados nas famílias Golenkiniaceae e Micractiniaceae (Chlorophyceae) que ocorrem no PEFI; e 2) apresentar descrições e ilustrações dos materiais identificados verificando novas ocorrências para o PEFI.

\section{Material e métodos}

O material planctônico foi coletado com rede confeccionada com tecido de náilon de $20 \mu \mathrm{m}$ de abertura de malha. As algas do perifíton foram coletadas manualmente mediante espremido de plantas submersas ou de partes submersas de plantas emergentes, coleta de exemplares inteiros ou de partes submersas de macrófitas aquáticas, além do material que habita sobre pedras ou a camada superficial do sedimento. Foram examinadas 39 amostras das quais sete apresentaram exemplares das famílias estudadas.

A espécie que consta da literatura, mas que não foi reencontrada neste estudo teve seu nome citado na chave de identificação e no texto antecedido por um asterisco.

O sistema de classificação utilizado para os gêneros e as espécies de Golenkiniaceae e Micractiniaceae foi o de Komárek \& Fott (1983).

A sequência dos gêneros dentro de cada família e das espécies dentro de cada gênero seguiu a ordem alfabética.

\section{Resultados e Discussão}

Foram identificados dois gêneros pertencentes às Golenkiniaceae e outros dois às Micractiniaceae. Os quatro gêneros possuem a seguinte situação sistemática:

Classe Chlorophyceae

Ordem Chlorococcales sensu latu

Família Golenkiniaceae

Golenkinia

Polyedriopsis

Família Micractiniaceae

Golenkiniopsis

Micractinium

\section{GOLENKINIACEAE}

Células solitárias ou que formam colônias temporárias. As células podem ser tetraédricas, poliédricas ou esféricas, a maioria delas possui cerdas e, por vezes, estão envolvidas em mucilagem. O cloroplastídio ocupa posição parietal na célula e tem um pirenoide cercado por amido. A parede celular possui espinhos de diferentes comprimentos, os quais estão uniformemente distribuídos na superfície da célula ou localizados em determinados pontos. A reprodução se faz por zoósporos, hemizoósporos ou autósporos (Comas 1996).

Chave de identificação dos gêneros de Golenkiniaceae

1. Células esféricas Golenkinia

1. Células poliédricas ou piramidais ........ Polyedriopsis 


\section{Golenkinia Chodat}

Indivíduos unicelulares geralmente isolados. As células são esféricas, possuem parede celular com espinhos distribuídos irregularmente em toda superfície e um cloroplastídio poculiforme, com um pirenoide reniforme na base do plastídio.

Golenkinia radiata Chodat, Journal de Botanique 8: 305, pl. 3, fig. 1-24. 1894.

Figuras 1-2

Células isoladas, esféricas, 10,0-15,0 $\mu \mathrm{m}$ diâm.; numerosos espinhos delicados, dispostos irregularmente na parede celular, 20,0-40,0 $\mu \mathrm{m}$ compr.; cloroplastídio poculiforme, 1 pirenoide reniforme. Autósporos formando agrupamentos temporários, frequentemente com 4 células, 4,7-5,3 $\mu \mathrm{m}$ diâm.

Hábitat: planctônico e perifítico.

Material examinado: BRASIL. São Paulo, São Paulo, Lago das Garças, 8-III-2007, S. Fernandes s.n. (SP390899).

Distribuição no PEFI: Lago das Garças (Sant'Anna et al. 1989); Lago do IAG (Ferragut et al. 2005).

O presente material concorda plenamente com o descrito em Sant'Anna et al. (1989). A espécie é facilmente confundida com Golenkiniopsis solitaria (Koršikov) Koršikov, mas é possível distingui-las pela forma do pirenoide, que em Golenkinia radiata Chodat é reniforme e em G. solitaria (Koršikov) Koršikov anelar. A espécie também foi registrada em trabalhos ecológicos realizados com material do PEFI por Bicudo et al. (1999) e Barcelos (2003) para o Lago das Garças e Biesemeyer (2005) para o Lago das Ninféias.

\section{Polyedriopsis Schmidle}

Indivíduos unicelulares de vida livre, em geral isolados, raro agrupados. A célula tem forma de almofada (todos os ângulos num mesmo plano) ou um tanto piramidal (ângulos em planos distintos), com quatro ou cinco ângulos levemente acuminados, retos ou, comumente obtusos, cada qual ornado com um a 10 espinhos delicados, que afilam gradualmente para o ápice. O cloroplastídio é único por célula, laminar e colocado lateralmente. $O$ pirenoide é, em geral, único e situa-se lateralmente na célula (Bicudo \& Menezes 2006).
Polyedriopsis spinulosa (Schmidle) Schmidle, Biologisches Zentralblatt5(1): 17.1899 $\equiv$ Tetraëdron spinulosa Schmidle, Allgemeine botanischen Zeitschrift 2: 193, fig. 2. 1896.

Figura 3

Células isoladas poligonais, piramidais, ca. 20,0 $\mu$ m diâm.; 4 ângulos, 3 espinhos por ângulo, ca. 13,0 $\mu \mathrm{m}$ compr.; cloroplastídio único, 1 pirenoide.

Hábitat: planctônico.

Material examinado: BRASIL. São PAulo, São Paulo, Lago das Garças, 8-III-2007, S. Fernandes s.n. (SP390899).

Distribuição no PEFI: Lago das Garças (Sant'Anna et al. 1989, Tucci et al. 2006).

Polyedriopsis spinulosa (Schmidle) Schmidle é a única espécie descrita até hoje para este gênero. Segundo Fernandes \& Bicudo (2009), é de pronto reconhecimento pela forma de almofada de suas células, cujos ângulos são ornados com tufos de três espinhos cada um.

\section{MICRACTINIACEAE}

Indivíduos isolados ou em colônias formadas por um a até vários grupos de quatro células cada um. As células são esféricas e possuem parede celular com espinhos dispostos radialmente. O cloroplastídio é único, parietal, poculiforme ou, mais raro, podem ser vários, com a forma de placas parietais. Um pirenoide pode ou não estar presente. A multiplicação assexuada ocorre por autósporos ou zoósporos e a reprodução sexuada por oogamia (Sant'Anna 1984).

\section{Chave para identificação dos gêneros de} Micractiniaceae

1. Organismos unicelulares isolados ...... Golenkiniopsis 1. Organismos formando colônias Micractinium

\section{Golenkiniopsis Koršikov}

Indivíduos unicelulares geralmente isolados; célula esférica, revestida por espinhos longos e delicados; cloroplastídio único, com um pirenoide anelar.

Chave para identificação das espécies de Golenkiniopsis

1. Células com espinhos longos, $>40 \mu \mathrm{m}$ compr. ...... G. longispina

1. Células com espinhos curtos, $<30 \mu \mathrm{m}$ compr. ....... G. solitaria 
Golenkiniopsis longispina (Koršikov) Koršikov, Viznacnik prisnovodnichi vodorostej Ukrainskoj RSR 5:265. 1953 EGolenkinia longispina Koršikov, Proceedings of the KharkovA. Gorky StateUniversity 10: 129. 1937.

\section{Figura 4}

Células isoladas, esféricas, ca. 12,3 $\mu \mathrm{m}$ diâm.; numerosos espinhos delicados, longos, extremidades ligeiramente arqueadas, dispostos regularmente na parede celular, 45,5-47,8 $\mu \mathrm{m}$ compr.; cloroplastídio poculiforme, 1 pirenoide anelar.

Hábitat: planctônico.

Material examinado: BRASIL. São PAULO, São Paulo, Lago das Garças, 16-VII-1998, A. Tucci \& C.L. Sant'Anna s.n. (SP365423).
Distribuição no PEFI: Lago das Garças (Tucci et al. 2006).

A espécie pode ser facilmente confundida com Golenkinia radiata Chodat, cuja distinção se faz pela forma do pirenoide, como mencionado anteriormente.

Golenkiniopsis solitaria (Koršikov) Koršikov, Viznacnik prisnovodnichi vodorostej Ukrainskoj RSR 5:249. 1953 E Golenkinia solitaria Koršikov, Proceedings of the KharkovA. Gorky State University 10: 135.1937.

\section{Figura 5}

Células isoladas, esféricas, 7,1-11,0 $\mu \mathrm{m}$ diâm.; numerosos espinhos delicados, longos, extremidades ligeiramente arqueadas, dispostos regularmente na

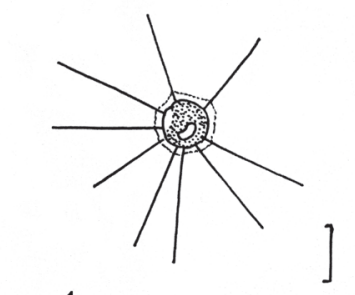

1
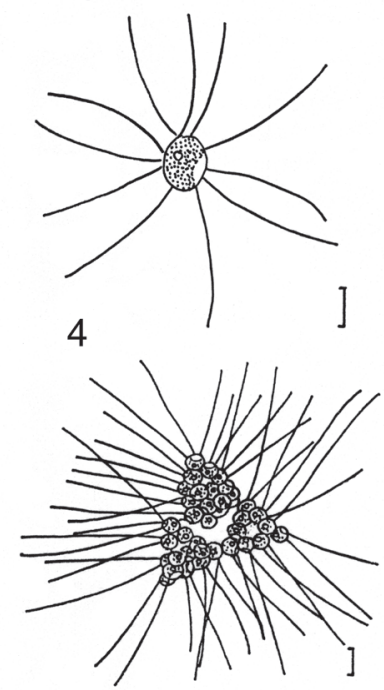

7

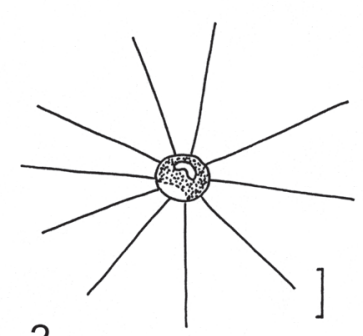

2

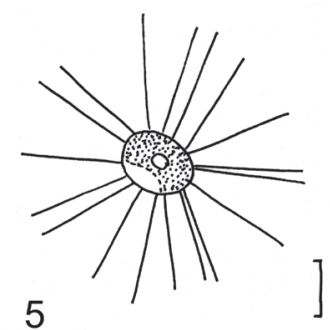

5

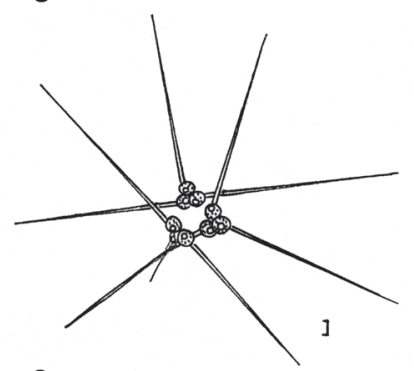

8
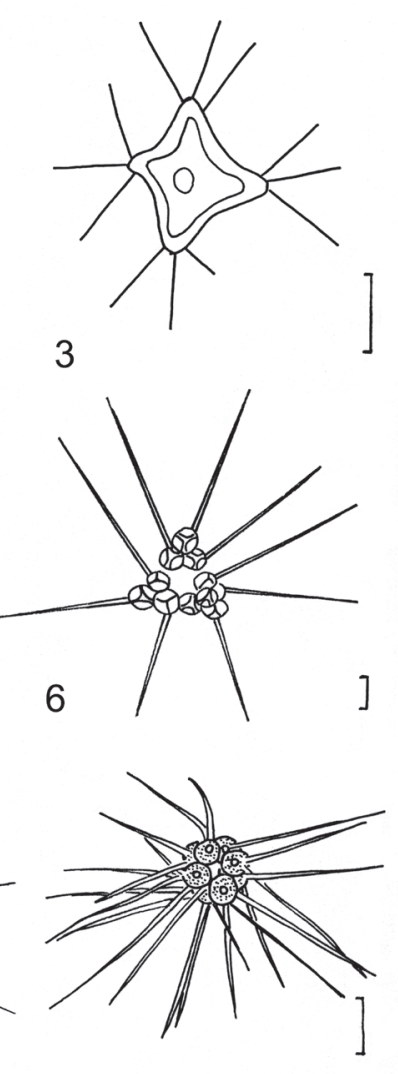

9

Figuras 1-9. Golenkiniaceae e Micractiniaceae do Parque Estadual das Fontes do Ipiranga, São Paulo, SP, Brasil. 1. Golenkinia radiata: detalhe do pirenoide reniforme. 2. Golenkinia radiata. 3. Polyedriopsis spinulosa (segundo Sant'Anna et al. 1989). 4. Golenkiniopsis longispina (segundo Tucci et al. 2006). 5. Golenkiniopsis solitaria. 6. Micractinium bornhemiense: auto-esporulação. 7. Micractinium bornhemiense (segundo Sant'Anna et al. 1989). 8. Micractinium crassisetum (segundo Sant'Anna et al. 1989). 9. Micractinium pusillum. Escalas $=10 \mu \mathrm{m}$.

Figures 1-9. Golenkiniaceae and Micractiniaceae from the Parque Estadual das Fontes do Ipiranga, São Paulo, São Paulo State, Brazil. 1. Golenkinia radiata: detail of the kidney-shaped pyrenoid. 2. Golenkinia radiata. 3. Polyedriopsis spinulosa (from Sant'Anna et al. 1989). 4. Golenkiniopsis longispina (from Tucci et al. 2006). 5. Golenkiniopsis solitaria. 6. Micractinium bornhemiense: autosporulation. 7. Micractinium bornhemiense (from Sant'Anna et al. 1989). 8. Micractinium crassisetum (from Sant'Anna et al. 1989). 9. Micractinium pusillum. Scales bar $=10 \mu \mathrm{m}$. 
parede celular, 16,9-28,0 $\mu \mathrm{m}$ compr.; cloroplastídio poculiforme, 1 pirenoide anelar.

Hábitat: planctônico.

Material examinado: BRASIL. São PaUlo, São Paulo, PEFI, Lago das Garças, 16-II-1998, A. Tucci \& C.L. Sant'Anna s.n. (SP365423); Lago dos Bambus, 23-IV-2012, N.R.M. Bento \& A. Tucci s.n. (SP427738).

Distribuição no PEFI: Lago das Garças (Tucci et al. 2006).

As duas espécies de Golenkiniopsis, G. longispina (Koršikov) Koršikov e G. solitaria (Koršikov) Koršikov, diferem entre si pelo diâmetro celular, que é maior em G. longispina (Koršikov) Koršikov e menor em G. solitaria (Koršikov) Koršikov, bem como, pelo comprimento do espinho, maior em G. longispina. Esta espécie foi mencionada em trabalho de ecologia realizado por Fonseca (2005) para o Lago das Garças.

\section{Micractinium Fresenius}

Colônias esféricas a triangulares e/ou piramidais, formadas por células esféricas a elipsoidais. Cada célula possui de um até vários espinhos periféricos longos e delicados. O cloroplastídio é único por célula, poculiforme e possui um pirenoide.

Chave para identificação das espécies de Micractinium

1. Células com espinhos cônicos, expandidos na base *M. crassisetum

1. Células com espinhos delgados, não expandidos na base

2. Colônias com arranjo piramidal das células ...... M. bornhemiense

2. Colônias com arranjo globular ou quadrático das células M. pusillum

Micractinium bornhemiense (Conrad) Koršikov, Viznacnik prisnovodnichi vodorostej Ukrainskoj RSR 5: 401.1953 Errerella bornheimiensis Conrad, Bulletin de la Société Botanique de Belgique 52:242, fig. 1-3. 1913.

Figuras 6-7

Colônias piramidais, formadas por 16-64 células; células esféricas, 5,0-6,0 $\mu \mathrm{m}$ diâm., 1-2 espinhos retos, hialinos, 30,0-45,0 $\mu \mathrm{m}$ compr., cloroplastídio poculiforme, 1 pirenoide. Autósporos presentes.

Hábitat: planctônico.
Material examinado: BRASIL. São Paulo, São Paulo, Lago das Garças, 16-II-1998, A. Tucci \& C.L. Sant'Anna s.n. (SP365418); 18-III-1998, A. Tucci \& C.L. Sant'Anna s.n. (SP365419); 15-IV-1998, A. Tucci \& C.L. Sant'Anna s.n. (SP365420); 16-IX-1998, A. Tucci \& C.L. Sant'Anna s.n.(SP365425); Lago dos Bambus, 23-IV-2012, N.R.M. Bento \& A. Tucci s.n. (SP427738).

Distribuição no PEFI: Lago das Garças (Sant'Anna et al. 1989, Tucci et al. 2006); Lago dos Bambus.

Espécimes de Micractinium bornhimiense foram registrados em processo de reprodução, autoesporulação (figura 6). Micractinium pusillum e M. bornhimiense assemelham-se bastante, porém, a última difere da primeira por apresentar disposição sempre piramidal das células nas colônias e espinhos mais longos (Sant'Anna 1984).

* Micractinium crassisetum Hortobágyi, Acta BotanicaAcademiaeScientiarumHungaricae 18: 123, 129, fig. 13. 1973.

Figura 8

Colônias com 4-8 células; células esféricas, 6,5-7,0 $\mu$ m diâm., 2-3 espinhos retos, 1,5-1,8 $\mu$ m diâm. na base, 30,0-40,0 $\mu \mathrm{m}$ compr.; cloroplastídio parietal, 1 pirenoide.

Hábitat: planctônico.

Distribuição no PEFI: Lago das Garças (Sant'Anna et al. 1989).

Material examinado: não detalhado em Sant'Anna et al. (1989).

Apesar da análise de amostras provenientes especialmente do Lago das Garças, a espécie não foi reencontrada naquelas atualmente examinadas. Sant'Anna et al. (1989) apresentaram ilustrações e medidas dos exemplares que identificaram, porém, não informaram os números de acesso dos materiais examinados no herbário, tornando impossível seu reestudo.

Micractinium pusillum Fresenius, Abhandlungen der Senckenbergische Naturforschende Gesellschaft 2: 236.1858.

Figura 9

Colônias globosas ou quadráticas, formadas por grupos de 4-8-16 células; células esféricas, 4,2-7,0 $\mu \mathrm{m}$ diâm., 3-5 espinhos retos, hialinos, 11,0-40,7 $\mu \mathrm{m}$ compr.; cloroplastídio poculiforme, 1 pirenoide. 
Hábitat: planctônico.

Material examinado: BRASIL, São Paulo. São Paulo, Lago das Garças, 16-II-1998, A. Tucci \& C.L. Sant'Anna s.n. (SP365418); 18-III-1998, A. Tucci \& C.L. Sant'Anna s.n. (SP365419); 15-IV-1998, A. Tucci \& C.L. Sant'Anna S.n. (SP365420); 16-IX-1998, A. Tucci \& C.L. Sant'Anna s.n. (SP365425); Lago dos Bambus, 23-IV-2012, N.R.M. Bento \& A. Tucci s.n. (SP427738).

Distribuição no PEFI: Lago das Garças (Sant'Anna et al. 1989, Tucci et al. 2006).

Os espécimes de $M$. pusillum Fresenius assemelham-se aos de M. bornhemiense (Conrad) Koršikov, porém, segundo Nogueira (1991), as colônias na primeira espécie apresentam arranjo das células geralmente tetraédrico ou com vários grupos tetraédricos de células reunidos, originando um arranjo quadrático ou poliédrico, cada célula com dois até oito espinhos hialinos. Difere também de $M$. bornhemiense (Conrad) Koršikov porque esta espécie apresenta arranjo piramidal das células na colônia e número reduzido de espinhos por célula. A espécie foi registrada em trabalhos de cunho ecológico realizados por Fonseca (2005) a partir de material do Lago das Garças e Biesemeyer (2005) do Lago das Ninféias.

Chave para identificação dos gêneros e espécies de Golenkiniaceae e Micractiniaceae do PEFI

1. Organismos formando colônias ..........(Micractinium)

2. Células com espinhos cônicos, expandidos na base. *M. crassisetum

2. Células com espinhos finos, não expandidos na base

3. Colônias com arranjo piramidal das células ..... M. bornhemiense

3.Colônias com arranjo globular ou quadrático das células M. pusillum

1. Organismos unicelulares, isolados

4. Células poliédricas ou piramidais

4. Células esféricas

5.Pirenoide reniforme .... Golenkinia (G. radiata)

5.Pirenoide anelar (Golenkiniopsis)

6. Células com espinhos longos, $>40 \mu \mathrm{m}$ de compr. G. longispina

6. Células com espinhos curtos, $<30 \mu \mathrm{m}$ de compr. G. solitaria

As duas famílias foram pouco representadas na área do PEFI, ocorrendo em quatro ambientes distintos, todos artificiais (reservatórios). O Lago das Garças foi o ambiente em que o maior número de espécies foi registrado, isto é, todas as identificadas neste estudo. A riqueza de espécies já foi bem estudada no Lago das Garças, como estudos de flora realizados por Sant'Anna et al. (1989) e Tucci et al. (2006), além de estudos ecológicos (ex. Sant'Anna et al. 1997, Tucci \& Sant'Anna 2003, Crossetti \& Bicudo, 2008, Gentil et al. 2008, Fonseca \& Bicudo 2011). Golenkinia radiata Chodat, Golenkiniopsis longispina (Koršikov) Koršikov, Micractinium crassisetum Hortobágyi e M. pusillum Fresenius ocorreram em dois ambientes cada um, enquanto que as demais três espécies o fizeram em um único. Todas as sete espécies neste trabalho foram coletadas no plâncton e Golenkinia radiata Chodat também no perifíton.

As famílias Golenkiniaceae e Micractiniaceae apresentam indivíduos, em geral, muito parecidos uns com os outros, o que pode dificultar sua identificação taxonômica. Por exemplo, de acordo com Koršikov (1953), Golenkinia difere morfologicamente de Golenkiniopsis apenas pela forma do pirenoide, que em Golenkinia é reniforme e em Golenkiniopsis anelar. Contudo, nem sempre a observação da forma do pirenoide é precisa e, muitas vezes, nem é possível. Há espécimes cuja forma do pirenoide esteve no limite entre o anelar e o reniforme e a decisão por uma ou outra forma tornou-se extremamente subjetiva. Também, visualizar a presença e a forma do pirenoide nos espécimes constituintes das falsas colônias foi bastante difícil. No entanto, quando visível, a identificação foi conclusiva. A presença de uma tênue camada de mucilagem envolvendo a base dos espinhos de Golenkinia e sua ausência em Golenkiniopsis seria outra forma de diferenciar os representantes desses dois gêneros quanto à morfologia, porém, nem sempre a detecção desse envoltório foi possível. A melhor diferença entre esses dois gêneros reside, então, na forma de reprodução, que em Golenkiniopsis se faz por zoósporos tetraflagelados ou oogamia, e em Golenkinia por zoósporos biflagelados e não apresentariam reprodução sexuada. Esta diferenciação é, entretanto, problemática, pois a observação de zoósporos biflagelados em Golenkinia ainda precisa ser confirmada.

Bicudo \& Menezes (2006) mencionaram que, raramente, os indivíduos-filhos de Golenkinia e Golenkiniopsis se mantêm juntos por conta do emaranhado de seus espinhos, formando agrupamentos que a literatura chama de falsas colônias. Mas, no material presentemente examinado foi possível 
encontrar, com certa frequência, vários indivíduos formando tais colônias falsas, ou seja, autósporos que permaneceram juntos temporariamente após a liberação da parede da célula-mãe. Assim, os espécimes com quatro células foram considerados resultados de autosporulação de Golenkinia ou Golenkiniopsis.

\section{Agradecimentos}

NRMB agradece ao Conselho Nacional de Desenvolvimento Científico e Tecnológico (CNPq), pela bolsa de Iniciação Científica; CR pela bolsa de Mestrado concedida pelo CNPq ao Programa de Pós-graduação em Biodiversidade e Meio Ambiente do Instituto de Botânica; e CEMB também ao CNPq, por Bolsa de Produtividade (Proc. No 309474/2010-8).

\section{Literatura citada}

Barcelos, E.M. 2003. Avaliação do perifíton como sensor da oligotrofização experimental em reservatório eutrófico (Lago das Garças, São Paulo). Dissertação de Mestrado, Universidade Estadual Paulista, Rio Claro.

Bicudo, C.E.M. \& Menezes, M. 2006. Gêneros de algas de águas continentais do Brasil: Chave para identificação e descrições. 2 ed. RiMa Editora, São Carlos.

Bicudo, C.E.M., Ramírez, R.J.J., Tucci, A. \& Bicudo, D.C. 1999. A dinâmica de populações fitoplanctônicas em ambiente eutrofizado: o Lago das Garças, São Paulo. In: Henry, R. (ed.). Ecologia de reservatórios: estrutura, função e aspectos sociais. Botucatu: FUNDIBIO/ FAPESP. pp. 449-508.

Bicudo, C.E.M., Ferragut, C. \& Massagardi, M.R. 2009. Cryptophyaceae population dynamics in an oligomesotrophic reservoir (Ninféias pond) in São Paulo, southeast Brazil. Hoehnea 36: 99-111.

Biesemeyer, K.F. 2005. Variação nictemeral da estrutura e dinâmica da comunidade fitoplanctônica em função da temperatura da água nas épocas de seca e chuva em reservatório urbano raso mesotrófico (Lago das Ninféias), Parque Estadual das Fontes do Ipiranga, São Paulo. Dissertação de Mestrado, Instituto de Botânica, São Paulo.

Buchheim, M., Buchheim, J., Carlson, T., Braband, A., Hepperle, D., Krienitz, L., Wolf, M. \& Hegewald, E. 2005. Phylogeny of the Hydrodictyaceae (Chlorophyceae): inferences from rDNA data. Journal of Phycology 41: 1039-1054.

Comas, G.A. 1996. Las Chlorococcales dulciacuícolas de Cuba. In: L.K. Hamburg \& S. Giessen (eds.). Blibliotheca Phycologica. Sttutgart, Gustav Fisher Verlag.
Comas, A. \& Sánchez, P. 2008. Algunas consideraciones y sugerencias sobre "la crisis en la taxonomía tradicional" con especial referencia a las algas verdes unicelulares (cocales). Boletín Sociedad Española de Ficologia: Algas 39: 16-20.

Crossetti, L.O. \& Bicudo, C.E.M. 2005. Effects of nutrient impoverishment on phytoplankton biomass: a mesocosms experimental approach in a shallow eutrophic reservoir (Garças pond), São Paulo, southeast Brazil. Revista Brasileira de Botânica 28: 95-108.

Crossetti, L.O. \& Bicudo, C.E.M. 2008. Phytoplankton as a monitoring tool in a tropical urban shallow reservoir (Garças Pond): the assemblage index application. Hydrobiologia 610: 161-173.

Fernandes, S. \& Bicudo, C.E.M. 2009. Criptógamos do Parque Estadual das Fontes do Ipiranga, São Paulo, SP. Algas, 26: Chlorophyceae (famílias Chlorococcaceae e Coccomyxaceae). Hoehnea 36: 173-191.

Ferragut, C., Lopes, M.R.M., Bicudo, D.C., Bicudo, C.E.M. \& Vercellino, I.S. 2005. Ficoflórula perifítica e planctônica (exceto Bacillariophyceae) de um reservatório oligotrófico raso (Lago do IAG, São Paulo). Hoehnea 32: 137-184.

Fonseca, B.M. 2005. Diversidade fitoplanctônica como discriminador ambiental em dois reservatórios rasos com diferentes estados tróficos no Parque Estadual das Fontes do Ipiranga, São Paulo, SP. Tese de Doutorado, Universidade de São Paulo, São Paulo.

Fonseca, B.M. \& Bicudo, C.E.M. 2008. Phytoplankton seasonal variation in a shallow stratified eutrophic reservoir (Garças Pond, Brazil), Hydrobiologia 600: 267-282.

Fonseca, B.M. \& Bicudo, C.E.M. 2011. Phytoplankton seasonal and vertical variations in a tropical shallow reservoir with abundant macrophytes (Ninféias Pond, Brazil). Hydrobiologia 665: 229-245.

Gentil, R.C., Tucci, A. \& Sant'Anna, C.L. 2008. Dinâmica da comunidade fitoplanctônica e aspectos sanitários de um lago urbano eutrófico em São Paulo, SP. Hoehnea 35: 265-280.

Hegewald, E., Hepperle, D., Wolf, M. \& Krienitz, L. 2001. Phylogenetic placement of Chlorotetraedron incus, C. polymorphum and Polyedriopsis spinulosa (Neochloridaceae, Chlorophyta). Phycologia 40: 399-402.

Komárek, J., \& Fott, B. 1983. Das Phytoplankton des Susswassers. Chlorophyceae (Grünalgen) Ordenung: Chlorococcales. E. Schweizerbart'sche verlagsbuchhandlung, Stuttgart.

Koršikov, A.A. 1953. Pidklas Protokokovi (Protococcineae). Viznacnik prisnovodnichi vodorostej Ukrainskoj RSR 5: $1-439$.

Krienitz, L. \& Bock, C. 2012. Present state of the systematics of planktonic coccoid green algae of inland waters. Hydrobiologia 698: 295-326. 
Krienitz, L., Hegewald, E., Hepperle, D. \& Wolf, A. 2003. The systematics of coccoid green algae: 18S rRNA gene sequence data versus morphology. Biologia, Bratislava, 58: 437-446.

Leliaert, F., Smith, D.R., Moreau, H., Herron, M.D., Verbruggen, H., Delwiche, C.F. \& Clerck, O.D. 2012. Phylogeny and Molecular Evolution of the Green Algae. Critical Reviews in Plant Sciences 31:1-46.

Lopes, M.R.M., Bicudo, C.E.M. \& Ferragut, C. 2005. Short term spatial and temporal variation of phytoplankton in a shallow tropical oligotrophic reservoir, southeast Brazil. Hydrobiologia 542: 235-247.

Nogueira, I.S. 1991. Chlorococcales sensu lato (Chlorophyceae) do município do Rio de Janeiro e arredores, Brasil: inventário e considerações taxonômicas. Dissertação de Mestrado, Universidade Federal do Rio de Janeiro, Rio de Janeiro.

Pröschold, T., Bock, C, Luo, W. \& Krienitz, L. 2010. Polyphyletic distribution of bristle formation in Chlorellaceae: Micractinium, Diacanthos, Didymogenes and Hegewaldia gen. nov. (Trebouxiophyceae, Chlorophyta). Phycological Research 58: 1-8.
Sant'Anna, C.L. 1984. Chlorococcales (Chlorophyceae) do Estado de São Paulo, Brasil. Bibliotheca Phycologica 67: 1-348.

Sant'Anna, C.L., Azevedo, M.T.P. \& Sormus, L. 1989. Fitoplâncton do Lago das Garças, Parque Estadual das Fontes do Ipiranga, São Paulo, SP, Brasil: estudo taxonômico e aspectos ecológicos. Hoehnea 16: 89-131.

Sant'Anna, C.L., Sormus, L., Tucci, A. \& Azevedo, M.T.P. 1997. Variação sazonal do fitoplâncton do Lago das Garças, São Paulo, SP. Hoehnea 24: 67-86.

Tucci, A. \& Sant'Anna, C.L. 2003. Cylindrospermopsis raciborskii (Woloszýnska) Seenayya \& Subba Raju (Cyanobacteria): variação semanal e relações com fatores ambientais em um reservatório eutrófico, São Paulo, SP, Brasil. Revista Brasileira de Botânica 26: 97-112.

Tucci, A., Sant'Anna, C.L. Gentil, R.C. \& Azevedo, M.T.P. 2006. Fitoplâncton do Lago das Garças, São Paulo, Brasil: um reservatório urbano eutrófico. Hoehnea 33: 147-175. 